\title{
A Case Study of Geochemical Exploration for Xishanwan Ag polymetallic deposit, Inner Mongolia, China
}

\section{Ming Kang ${ }^{1}$ Ye Yuan ${ }^{2}$}

1. School of Earth Science and Resources, Chang'an University, Xi'an, 710054, China; kangmin@chd.edu.cn 2. School of Earth Science and Resources, Chang'an

University, Xi'an, 710054, China; 380634072@qq.com

Xishanwan Ag polymetallic deposit is geotectonically situated in the midwestern section of the northern margin of the North China Craton. The host rocks of the silver ore body are mainly volcanic-subvolcanic rocks, and the host rocks of the lead-zinc ore body are mainly medium-acid intrusive rocks. The deposit was discovered by a set of geochemical exploration method using the "outlining the anomaly district in regional area, selecting the target from the anomaly district, and positioning from the target". In regional area , multielement anomalies of $\mathrm{Ag}, \mathrm{W}, \mathrm{As}, \mathrm{Au}, \mathrm{Sb}, \mathrm{Pb}, \mathrm{Bi}, \mathrm{Mo}, \mathrm{Zn}, \mathrm{Cu}$, $\mathrm{Hg}$ were outlined in Mazongshan area (MZS) through 1:50000 stream sediment survey. The anomalies occupy an area of about $24.3 \mathrm{~km}^{2}$, and the anomalies of $\mathrm{Ag}, \mathrm{As}, \mathrm{Sb}, \mathrm{W}$ and $\mathrm{Au}$ are consistent and continuous with clear peaks. The combined anomalies are related to the distribution of exposed volcanic rocks on the surface. In the MZS anomaly area, the Xishanwan target area (XSW) is preferred by $1 / 10,000$ soil geochemical measurement and lithogeochemical measurement. The specific positioning by the primary halo method of the trench in the XSW target.

The extraction model for geochemical anomaly thresholds based on genetic algorithm and spatial interpolation algorithm is proposed for the delineation of geochemical anomalies. The processing results for the genetic algorithm model is used mainly to reflect the background information, and the spatial interpolation model processing results comprehensively include background information and abnormal information. The distribution characteristics of the two sets of data are superimposed, and the intersection point can obtain the geochemical anomaly threshold value. The value can reflect the anomaly information truly and objectively, and the weak anomaly can be identified more sensitively, which can improve the effect of anomaly delineation. The discovery of the Xishanwan silver polymetallic deposit is an example.

Keywords: Ag polymetallic deposit; geochemical exploration; Xishanwan; Inner Mongolia 